\title{
Minimum Volume Discussion in the Treatment of Colon and Rectal Cancer: A Review of the Current Status and Relevance of Surgeon and Hospital Volume regarding Result Quality and the Impact on Health Economics
}

\author{
Karl-Heinrich Link ${ }^{a, b}$ \\ Ludger Staib ${ }^{\text {b,e }}$ \\ a Department of Surgery, Asklepios Paulinen Klinik, Wiesbaden, Germany; \\ ${ }^{b}$ Forschungsgruppe Onkologie Gastrointestinale Tumoren (FOGT), University of Ulm, Ulm, Germany; \\ ${ }^{c}$ Department of Health Economics, RheinMain University of Applied Sciences, Wiesbaden, Germany; \\ ${ }^{\mathrm{d}}$ Department of General and Visceral Surgery, University of UIm, UIm, Germany; \\ e Department of General and Visceral Surgery, Klinikum Esslingen, Esslingen, Germany
}

Peter Coy ${ }^{c}$ Mark Roitman ${ }^{a}$ Carola Link $^{b} \quad$ Marko Kornmann ${ }^{b, d}$

\section{Keywords}

Colon cancer · Rectal cancer · Quality · Surgeon volume · Hospital volume

\section{Summary}

Background: To answer the question whether minimum caseloads need to be stipulated in the German S3 (or any other) guidelines for colorectal cancer, we analyzed the current representative literature. The question is important regarding medical quality as well as health economics and policy. Methods: A literature research was conducted in PubMed for papers concerning 'colon cancer' (CC), 'rectal cancer' (RC), and 'colorectal cancer' (CRC), with 'results', 'quality', and 'mortality' between the years 2000 and 2016 being relevant factors. We graded the recommendations as 'pro', 'maybe', or 'contra' in terms of a significant correlation between hospital volume (HV) or surgeon volume (SV) and treatment quality. We also listed the recommended numbers suggested for $\mathrm{HV}$ or SV as minimum caseloads and calculated and discussed the socio-economic impact of setting minimum caseloads for CRC. Results: The correlations of caseloads of hospitals or surgeons turned out to be highly controversial concerning the influence of HV or SV on short- and long-term surgical treatment quality of CRC. Specialized statisticians made the point that the reports in the literature might not use the optimal biometrical analytical/reporting methods. A Dutch analysis showed that if a deci- sion towards minimum caseloads, e.g. $>50$ for CRC resections, would be made, this would exclude a lot of hospitals with proven good treatment quality and include hospitals with a treatment quality below average. Our economic analysis envisioned that a yearly loss of EUR $<830,000$ might ensue for hospitals with volumes $<50$ per year. Conclusions: Caseload (HV, SV) definitely is an inconsistent surrogate parameter for treatment quality in the surgery of $\mathrm{CC}, \mathrm{RC}$, or CRC. If used at all, the lowest tolerable numbers but the highest demands for structural, process and result quality in the surgical/interdisciplinary treatment of $\mathrm{CC}$ and RC must be imposed and independently controlled. Hospitals fulfilling these demands should be medically and socio-economically preferred concerning the treatment of $\mathrm{CC}$ and $\mathrm{RC}$ patients.

(c) 2017 S. Karger GmbH, Freiburg

\section{Introduction}

Colon cancer (CC) and rectal cancer (RC) are two different tumor entities with regard to many parameters [1]. The incidence of both entities is high in the Western countries (Europe and the USA/Canada) and increasing with age [2]. Besides different reasons of as well as mechanisms in causal and molecular carcinogen-

\section{KARGER}

(c) 2017 S. Karger GmbH, Freiburg
Prof. Dr. Dr. h.c. Karl-Heinrich Link 
esis [3] and prevention [1], CC and RC receive different multimodal therapies (CC: UICC IIB and III: adjuvant chemotherapy (adjCT); RC: UICC II + III up to $12 \mathrm{~cm}$ from the anal verge: neoadjuvant radiochemotherapy (neoRCT) and postoperative adjuvant CT (neoRCT + adjCT) [4]. The surgical treatment for cure is different for CC and RC, although the surgical principles in CC and $\mathrm{RC}$ include resection within the embryonal sheaths (mesocolon and mesorectal fascias). However, surgery of RC in the pelvis by total mesorectal excision or partial mesorectal excision, sphincterpreserving abdominoperineal resection, or total abdominoperineal extirpation, all with nerve preservation, require special surgicalanatomical knowledge and expertise. The local relapse rates have been higher in RC, metastatic progression after surgery for cure differs in the location of the primary metastatic target organ $[5,6]$, and cure rates after multimodal therapy for RC have been inferior to CC, although stage-adapted 5-year survival results of CC and $\mathrm{RC}$ resected for cure are approaching each other [2]. Short-term results for morbidity and mortality also differ between CC and RC [6]. Obviously, the quality regarding short- and long-term results differs among surgeons and hospitals $[7,8]$, but many decision makers assume that there is a strict correlation between surgeon volume (SV) or hospital volume (HV) and outcome of quality. Most of the proponents of the strict correlation between SV and/or $\mathrm{HV}$ and result quality demand regulations and a shift of CC and RC patients to high-volume surgeons (HVS) or high-volume hospitals $(\mathrm{HVH})$. From our point of view [8], this regulative procedure might have disadvantages for the patients' safety and comfort and will have an unjustifiable negative impact on health economics. At the time when some surgeons demanded 50 cases (for CC and RC each) per year for surgeons as well as hospitals as minimum volumes for German large bowel cancer centers (LBCC), we pointed out in a critical review, with many surgeons from various institutions/societies partaking as coauthors, that caution must be exercised when HVS or HVH are only required as surrogate parameters for optimal treatment quality [8]. However, many medical (and health) policy experts today are still suggesting minimum volumes as the only surrogate parameter for high quality. Since we proposed nine requirements for surgeons or hospitals as the basis for high quality (including relatively low volumes) [7, 8], we were eager to discover whether the evidence has changed solely in favor of volume and reviewed the current literature between 2000 and 2016 to find out what should be recommended to surgeons and hospital managements in terms of structures providing optimal quality results of CC and RC treatment. We also analyzed the economic impact on hospital care in general, if decisions were made towards high volumes for surgeons and/or hospitals, which are not fully evidence-based. The results with new substantial and highly controversial data based on the literature are striking and confirm our statement from 2010, namely that volume only is not a reliable surrogate parameter for surgical oncological quality [8].

\section{Methods}

We reviewed the international literature regarding the topics CC, RC, and colorectal cancer (CRC), HV and SV, 'mortality', 'results', and 'quality' by researching the PubMed database and considering papers written in English or in German (with English abstracts). The literature contributions were made from various continents, countries, counties, and societies. The authors were either surgeons or health economists or statisticians. The literature reports were based on Cochrane databases, meta-analyses of reports on the topic, pooled analyses, or single-institution analyses. We analyzed the papers according to their final statements on the correlation between SV and/or HV and short-term (hospital morbidity and/or mortality) and/or long-term (survival) quality in CC, RC, and CRC with either 'pro' (depicted in green), 'maybe' (yellow), or 'contra' (red) volume as surrogate parameter for quality (fig. 1). Short-term quality was usually related to hospital mortality after surgery. The impact of volume on the quality of the other applied oncologic treatment modalities, i.e. RCT or CT, was mentioned in none of the papers. The strength of the literature statements was related to the total patient volumes analyzed and depicted by means of the size of encircled citations placed in the figures for CC, RC, and CRC (fig. 1). We refrained from using box plots. Furthermore, we listed the ranges of minimum volumes demanded by the various authors or national societies for CC, RC, and CRC in tables. Based on these results and by elucidating important papers, we generated the base for our conclusion by means of discussion, thus including suggestions for future decisions on the definition and provision of treatment quality in CC and RC. To make a statement on the socio-economic impact of guiding a multitude of patients with CC or RC to a limited number of surgeons or hospitals in a county or country, we also analyzed the literature available on that topic from 2000-2016.

\section{Results}

Correlation of Volumes to Treatment Quality in Colon Cancer, Rectal Cancer and Colorectal Cancer

The statements on whether HVS or HVH show significantly better results than non-HVS or non-HVH regarding short-term quality (mortality) or long-term results (long-term survival) in CC, $\mathrm{RC}$, or CRC are summarized in figure 1 . The treatments included in part multimodal therapy.

\section{Colon Cancer}

In CC, two authors each support a positive correlation between volume and treatment quality in favor ('pro') of either $\operatorname{HVH}[9,10]$ or HVS $[11,12]$ with respect to short-term outcome. However, two of these papers also come to the conclusion that the impact of volume on outcome 'may be' positive when correlated to HVH [11, 12] (these authors found a positive correlation for HVS). The estimation 'maybe' is made by one group only for the category HVS 
Short-term quality (mortality) better?
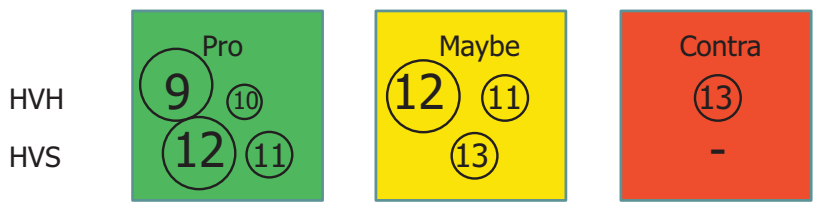

Long-term oncological results (OAS) better?

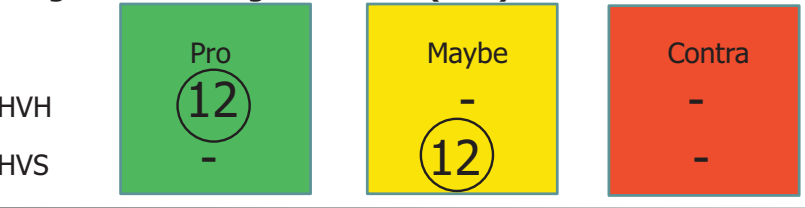

b Short-term quality (mortality) better?

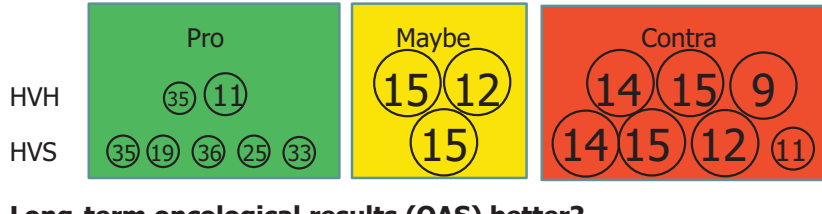

Long-term oncological results (OAS) better?

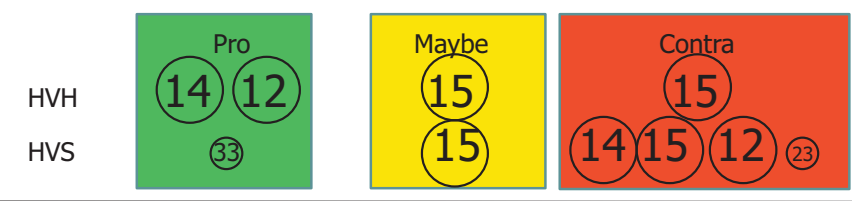

C Short-term quality (mortality) better?
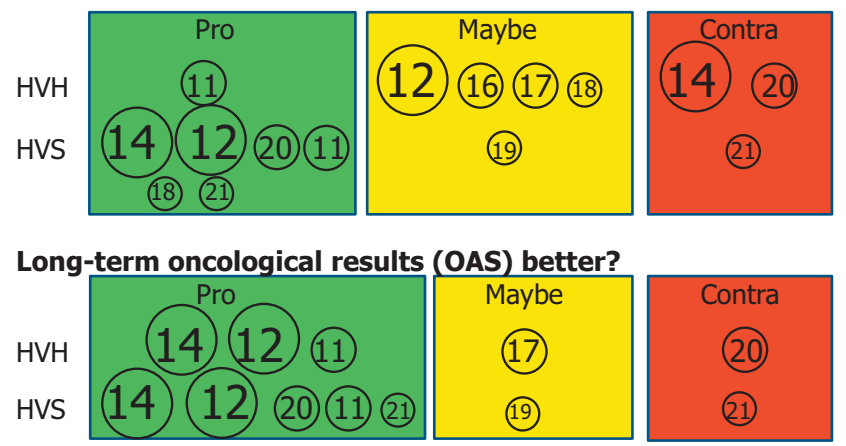

Fig. 1. The statement of the authors on the relevance of volume for treatment quality in CC (a), RC (b), and CRC (c) are depicted as 'pro' (green), 'maybe' (yellow), or 'contra' (red) for hospital volume (HV) or surgeon volume (SV). The sizes of the circles are representative for the number of patients analyzed in the cited papers. The paper cited is given as the reference number (from the reference list) within the circles: $\mathrm{n} 1>100,000, \mathrm{n} 2=10,000-100,000$, and $\mathrm{n} 3<$ 10,000 .

[13], but not for HVH. These authors reject a correlation between volume and quality for HVH. Only two statements are given concerning the correlation of volume to long-term results, one with 'pro' for HVH but 'maybe' for HVS [12]. Another group gives an ambiguous statement for HVS [14]. The authors make the statement that their results confirm the presence of a volume-outcome relationship in CRC surgery, based on 'hospital and surgeon case load, and benefits of specialization'. For death within 5 years of treatment, HV appeared to be more beneficial in RC surgery than for CC [14]. No paper rejects a correlation between volume and outcome concerning short-term outcome of HVS or long-term results and HVH or HVS (fig. 1a). None of the papers finds a positive correlation of both HVH and HVS to short- or long-term quality in the treatment of CC.

\section{Rectal Cancer}

More papers have dealt with correlations of volume and quality in RC surgery. A positive ('pro') correlation of HVH or HVS to overall short- and long-term treatment quality is reported by 8 reports. One group states that there 'may be' or that there is even no ('contra') correlation between volume and quality regarding either HVH or HVS [15]. No correlation between volume and quality as to short- and long-term results in HVH or HVS is reported in 6 papers. 'Pro' and 'contra' are nearly balanced in the evaluation of volume influence on outcome in RC.

\section{Colorectal Cancer}

When evaluating the impact of volume on outcome in CRC, 6 groups state that there is a positive correlation and one group [11] finds a definite association between short- and long-term outcome as to both HVH and HVS. Doubt ('maybe') is expressed as to the correlations of HVH $[12,16-18]$ or HVS [19] to short-term quality and regarding long-term quality in HVH [17] or HVS [19]. Three authors reject correlations $[14,20,21]$. Among these, one group finds that $\mathrm{HVH}$ is not associated with quality [14] but that, on the contrary, HVS show better quality in short- and long-term outcomes [14]. Again, the statements 'pro', 'maybe', or 'contra' are far from being concordant.

\section{Suggested Minimum Volumes in Colon Cancer, Rectal Cancer and Colorectal Cancer}

Several national societies or various authors have defined minimum volumes necessary for higher quality in CC, RC or CRC treatment either for hospitals (table 1) or for surgeons (table 2).

When looking at the patient numbers which are discussed or suggested as minimum volumes recommended for hospitals (HV) assumed to be of higher quality in CC, RC or CRC treatment, a wide variety is given either in the recommendations of national cancer societies or by individual authors (table 1). For CC, these numbers range between 28 and 126 patients per year which ought to be surgically treated; the corresponding patient number range is 7-25 for RC and 21-150 for CRC.

A wide range of minimum patient numbers is given if minimum caseload for surgeons (SV) was defined (table 2), i.e. 4-11 for CC, 5-10 for RC, and 3.25-50 for CRC.

\section{Volume and Health Economics in the Treatment Strategy for Colon Cancer, Rectal Cancer and Colorectal Cancer}

Minimum volume definitions for hospitals or surgeons might have a severe economic impact since, for the time being, only the 
Table 1. Minimum volumes defined per 'hospital volume' (HV) (cases per hospital and year)

\begin{tabular}{llll}
\hline & $\begin{array}{l}\text { Colon } \\
\text { cancer }\end{array}$ & $\begin{array}{l}\text { Rectal } \\
\text { cancer }\end{array}$ & $\begin{array}{l}\text { Colorectal } \\
\text { cancer }\end{array}$ \\
\hline National & & & \\
Netherlands & - & 20 & 50 \\
Germany (DKG) & 40 & 20 & 60 \\
US (ACS) & - & - & 21 \\
\hline Authors & $28-126$ & $7-25$ & $21-150$ \\
\hline
\end{tabular}

Table 2. Minimum volumes defined for 'surgeon volume' (SV) (cases per surgeon and year)

\begin{tabular}{llll}
\hline & $\begin{array}{l}\text { Colon } \\
\text { cancer }\end{array}$ & $\begin{array}{l}\text { Rectal } \\
\text { cancer }\end{array}$ & $\begin{array}{l}\text { Colorectal } \\
\text { cancer }\end{array}$ \\
\hline $\begin{array}{l}\text { National } \\
\text { Germany (DKG) }\end{array}$ & - & 10 & - \\
\hline Authors & $4-11$ & $5-10$ & $3.25-50$ \\
\hline
\end{tabular}

Table 3. Parameters influencing the validity of analyses of volume-outcome correlations in colon and rectal cancer surgery

Biometry influences conclusions as to volume outcome [9, 16, 19, 22, 23] Differences in short- and long-term outcome are varying in their extent from 'sparse' to 'highly significant'

Variables/prognostically relevant factors other than biometrical methods with impacts on variation of results

Definition of 'rectal cancer', age, method calculating survival, method defining morbidity, surgical method (laparoscopy/open), population country, continent, various definitions of 'HVH' and 'HVS', definition of effect, SEER not representative for the 'whole' time periods analyzed, \% of emergency cases, validity of publication (impact factor of journal), postoperative mortality excluded in survival calculation, data from multimodal trials, question of analysis, number of patients analyzed, differences between hospitals, countries and continents.

minority of patients (CC $16.3 \%$, RC $27 \%$ ) is treated by so-called specialists in the USA. Additionally, in an analysis, the effect of specialization on treatment quality might be limited to subtypes of bowel cancer (RC UICC II) [22]. Shifting volumes to specialists may change referral practice and treatment convenience with influence on health economics.

The scientific basis of recommendations for minimum volumes has been severely criticized because of biometrical reasons. Several authors have stated that trials are very heterogeneous concerning patient and hospital characteristics (table 3) or are applying less adequate biometrical methods, altogether having a substantial impact on the outcome of a trial and, therewith, the conclusions $[9,16,19$, 22, 23].

A Dutch analysis found out that only a minority of hospitals with caseloads below the minimum HV (50 colorectal resections) were worse than average concerning morbidity ( $13 \%$ of all hospitals with caseloads <50/year) and mortality ( $8 \%$ of the hospitals with caseloads $<50 /$ year). 'Top quality' in both parameters of quality, i.e. morbidity and mortality, was delivered by $1 / 89$ hospitals only, and 'flop quality' in both parameters by only $1 / 89$ hospitals. The authors concluded that more parameters are required than only volume [16].
The authors also stated that cutoffs by volume might generate unjustified economic misbalances. We calculated the impact of these cutoffs on the 'low-volume but high-quality hospitals'.

We made a calculation in order to answer the question 'What would the economic impact be for the 155 hospitals with surgical departments in the state of Hesse' if the 'Dutch' definition of a minimum volume $>50 \mathrm{CC}$ and/or RC resections would be applied. Currently, only one third of these hospitals (52/155) would fulfill the minimum volume requirement. If a hospital is excluded from $\mathrm{CC}$ and RC treatment, it must be assumed that other large bowel surgical indications are subsequently reduced by change of referrals. If a hospital has $\leq 50 \mathrm{CRC}$ operations and $\leq 150$ other large bowel surgical interventions (e.g. diverticulitis, full-thickness excision of large polyps using minimally invasive procedures, inflammatory diseases, and so forth) which would be reduced by at least $50 \%$, the loss of income concerning CRC resections could be up to EUR 560,000, and for non-malignant colorectal surgical indications up to EUR 270,000, totaling EUR 830,000 per year. This exceeds more than $10 \%$ of the total income of a general/visceral surgical department in a medium-sized hospital.

\section{Discussion}

Volume discussions are frequently led with controversial arguments by the medical, political and health economic communities. At the time when LBCC were to be structured by experts of the German Cancer Society (GCS), one expert demanded 50 CC and $50 \mathrm{RC}$ per year as minimum volume for LBCC. Questioning this demand, which by our calculations could have been met by only 5-10 hospitals in Germany, we formed an expert group of leading German hospital surgeons to define the requirements, including volume, which should be fulfilled by surgeons to provide the highest possible treatment quality to their patients [8]. Our list of quality criteria, including volumes, has been an integrative part of the LBCC structure, and up to date more than 260 LBCC among a total of 1,018 organ centers have been established by the GCS [24]. Volumes still remain a point of discussion within the structural commission for LBCC certified and audited by the GCS and within various other decision making groups. So far, the minimum volumes required by the GCS (rectal cancer 20, colon cancer 30) are lower, but also higher, than those demanded by the literature reports cited in this paper.

In general, it is assumed by the medical and public community that the increase of caseload has a directly proportional impact on short-term (morbidity and mortality) and long-term quality (relapse-free and overall survival rates) in the surgical-oncologic treatment of CC and RC. We had stated that more interdisciplinary and structural parameters than merely minimum caseloads of surgeons or hospitals are defining treatment quality $[7,8]$. In many contemporary literature reports and in several national guidelines, however, defined minimum caseloads are the only parameter thought to be associated with treatment quality. We were eager to find out whether this assumption is justified and reviewed the cur- 
rent literature. If it would turn out to be not the case and if decisions would still stick to the demand for cutoffs of minimum volumes for either surgeons or hospitals, this could cause major unjustified frictions not only from the point of view of health care provision but also regarding the economic perspective of hospitals to be excluded. Therefore, in addition to generating scientific evidence for minimum volumes in terms of 'pro', 'maybe', or 'contra', we also calculated the financial impact of an exclusion of surgeons who are definitely qualified and/or of hospitals that do not fulfill the demands for minimum caseloads.

In our analysis, we found out that the definition of caseloads (SV and/or HV) is highly controversial with a wide range of suggestions or decisions and, if 'pro' was stated, of the recommended numbers for minimum caseloads (SV and/or HV). There is great variation in the conclusions of 'pro' (yes), 'maybe', or 'contra' (no) as to the demand for minimum volumes as surrogate parameter for quality in the surgical-oncologic treatment of CC, RC or CRC patients for surgeons (SV) or hospitals (HV). A respectable cohort of authors analyzing the impact of patient volume on quality came to very heterogeneous conclusions, with all of them thought to be evidence-based (fig. 1). In addition, the authors frequently gave different recommendations for SV or HV in CC, RC or CRC. Basically, when looking at the wide ranges of minimum 'patient load recommended' (table 1,2), doubts might be cast on the scientific basis for caseloads for SV or HV recommended for quality treatment of CC or RC. Our review is based on a PubMed research concerning publications on our topic during 2000 and 2016. The list, like other citation lists, might be incomplete, which could be interpreted as a methodological drawback. It is recognizable from several papers analyzed for our review that completeness in citation cannot always be achieved. Our figures and tables demonstrate the heterogeneity of opinions/statements which is unlikely to be changed by adding more authors (which in part have been included in our previous reports from 2009 [7] and 2010 [8]). Thus, there is clearly substantial and justified criticism of volume alone as surrogate parameter for quality. The prerequisites for quality by far exceed the single surrogate parameter 'volume', and quality has to be defined by composite markers.

Many groups have shown that other than caseloads, only training [25], hospital and team structure [26], and regular audits [16, 27] are important for providing and judging treatment quality. These measures should be implicated for warranting the best surgical-oncologic treatment quality. Kolfschoten et al. [16] reported that the Dutch Surgical Colorectal Audit (DSCA) checked 89 hospitals for their quality results in CRC surgery by the combined measure of volume and outcome ('serious' morbidity and postoperative mortality) (CM-V\&O)). When comparing the results before (2010) and after (2011) auditing, the values for mortality/morbidity were significantly reduced from $4.1 \%$ (mortality) and $24.3 \%$ (morbidity) in 2010 to $3.7 \%$ and $21.5 \%$ in 2011, respectively, upon examining data of 8,911 (2010) and 9,212 (2011) patients ( $\mathrm{p}<$ 0.01 ). The authors pointed out in 2014 that 'three years after the introduction of clinical auditing, a significant improvement in various process and outcome measures was observed, while variation in hospital performances decreased' [16]. In an analysis of our two large multimodal treatment trials for either CC (FOGT 1) or RC (FOGT 2) we found out that hospital size had no significant impact on quality results [6]. Caseload, e.g. according to a very large literature (meta-)analysis, had only a minor impact on treatment quality of RC patients [15], and in a most recent analysis of SEER data on CC (21,432 patients) and RC (5,893 patients) treatment, it was stated that volume is only associated with better quality in RC stage UICC II [22]. Together with the data of our review, these reports should generate critical reflections when decisions have to be made concerning minimum volumes for surgeons and/or hospitals. The deciding committees must also bear in mind that exceeding an upper limit of caseload might not lead to a gain but to a loss of lives, as shown in a Canadian analysis. While this Canadian study on the influence of HV on surgical outcome was able to show a benefit for increasing volumes in esophageal and pancreatic resections, this could not be shown for colorectal resections. In contrast, mortality was even higher in HVH [28].

Regarding the statement that most reports that try to define minimum caseloads are not even using the optimal and relevant statistical methods for their analyses, the frequent conclusion 'volume is the only predictor for quality' must be taken with even greater caution. Kim et al. [9] questioned the statement ' $70 \%$ of the studies found a volume effect in surgery of six cancer operations' made by Halm et al. [29] in 2002 after reviewing 130 volume outcome studies. The criticizing authors from the Departments of Economics, Mathematics, Public Policy, and Medicine at Rice University and Baylor College of Medicine in Houston, TX, USA, analyzed the patient-level hospital discharge data from three US states and data from the American Hospital Association Annual Survey of Hospitals from 2000-2011 [9]. First and foremost, they looked at the statistical methods suitable for such analyses and came to the surprising conclusion that the biometric method applied in most of the literature studies is inadequate [9]. In their analysis correlating patient outcome (mortality) and HV in 279,414 cancer patients who had resections of colon $(164,804)$, rectal $(36,046)$, esophageal, pancreatic and pulmonary malignant tumors, they came to the conclusion that quality in the resection of colon, pancreatic and pulmonary malignant tumors is significantly influenced by volume effects. Contrary to the logistic regression model, which is most commonly used in the literature, the authors defined models that control for time-invariant heterogeneity, such as the biometrical method of proven superiority. The critical influence of biometric methods on the results has also been recognized by other groups $[9,16,19,23]$. In our analysis, we did not use box plots generating average values to calculate a significance for 'pro' or 'contra', since such an important decision of making a recommendation to a patient where and by whom he has to receive $\mathrm{CC}$ or RC treatment should be made without doubts in the literature, namely without the numerous 'maybes' or 'contras' that we cited. The evaluation of the literature for decision making in a particular nation is even more complicated. Of course, when analyzing patient groups for correlations, the variable patient characteristics and analytical procedures must be taken into account when trying to make a relevant 
statement. In the USA, it is known that African-Americans have a different outcome than Caucasian Americans; European outcomes differ from the US results. Indeed, a large list of characteristics has an influence on the analytic results of short- and long-term outcome in the treatment of CC and RC (table 3).

Regarding our analysis, finding a great variability of 'pro', 'maybe' and 'contra' recommendations for volume effects, and with a huge variation of minimum numbers recommended for either CC or RC together with the profound criticism of experts [9] of the majority of publications concerning the volume effect in treatment quality of CC and RC, we come to the conclusion that volume alone as surrogate parameter for treatment quality in CC and RC is insufficient and no base for decisions concerning centralization. Therefore, the medical-political impetus to define minimum caseloads for surgeons and hospitals treating CC and/or RC patients has no basis of high evidence, and it must be stated that based on the result of these demands, namely the exclusion of surgeons or hospitals from treating those patients who prefer to be treated in a hospital easily to reach by themselves and their relatives, it is more than justified to look at the economic and social consequences which would be generated by driving patients to HVH/HVS.

For the DSCA, Kolfschoten et al. [16], as already mentioned above (validity of audits for quality improvement), also analyzed the economic consequences of setting 50 cases per year as minimum HV for 'colorectal resections' (cancer and benign indications). In their 2-year analysis (2010 and 2011) of morbidity and mortality in the subset of 18,123 patients with CRC treated in 89 hospitals (96\% of the Dutch hospitals), 61/89 hospitals with numbers lower than $50 /$ year would be excluded from treatment. Only 8/61 and 5/61 of the excluded hospitals were worse than average concerning morbidity (13\%) and mortality (8\%), respectively; vice versa, hospitals exceeding 50 cases per year but with worse quality than average would be included. They concluded that minimum volume alone in the Netherlands is not reliable to promise quality; low-volume hospitals (LVH) that are good in outcome (better than average) might be excluded, and, vice versa, HVH that are worse (than average) in outcome are included. If a decision would be made on centralization towards quality, the combined measure of 'volume' and 'outcome' (CM-V\&O) should be applied to select the 'qualified hospitals' [16].

Translated to the situation of a German medium-sized hospital with a general/visceral surgical department, the yearly loss of income could be up to EUR 830,000 according to our calculations if a minimum volume of $>50$ CRC resections would be politically required for a hospital that is thought to be of good quality for this indication. Medically and socio-economically, this political decision cannot be justified according to our data analysis.

When looking at the insecurity of the 'volume = quality' assumptions and with regard to the severe socio- and health economic consequences, we strongly demand that more must be taken into account than only volume. 'Volume only' as predictive parameter for treatment quality has been rejected in our previous reports $[7,8]$ and subsequently by other groups as well $[16,17]$. We had defined a list of quality parameters (table 4 ) to which 'regular independent audits' must now be added.
Table 4. Suggested structural measures/conditions for optimal structural, process and result quality in surgical-oncologic therapy of patients with colon or rectal cancer (modified from [7])

1. Respect current guidelines of national scientific societies (e.g. S3 guidelines 'Colorectal Cancer' of the German Cancer Society)

2. Interdisciplinarity in diagnosis and treatment (gastroenterology, intensive care, medical oncology, pathology, radiology, radiotherapy, surgery)

3. Decision making in regularly meeting interdisciplinary tumor boards

4. Diagnosis and treatment according to pathways

5. Responsible surgery or assistance by a 'visceral surgeon' (German qualification) or a surgeon with a comparable qualification (e.g. EBSQU-qualified coloproctology)

6. Organization of or participation in specialized seminars/congresses on the topic 'Colon and Rectal Cancer' at least once per year.

7. Minimal caseload per year and institution: colon cancer 20, rectal cancer 10.

8. Participation in quality assurance measures that document short- and longterm result quality

9. Participation in scientific trials

In this list, supported by the coauthors and the German surgical society 'Konvent Leitender Krankenhauschirurgen', we also defined the minimum volumes for hospitals, deduced from the reports up to our recent publication in 2010 [8]. Based on the very conflicting statements derived from our current literature analysis, the very lowest volume limits for 'colorectal surgery' could be suggested as follows: SV 4 CRC patients operated per year (3.5/year on average); HV 21 CRC patients operated per year (table 1, 2), in addition to the other quality structures of hospitals and the qualification of surgeons. As to the opinion of many German visceral surgeons qualified for CC and RC surgery (including the surgical authors of this review article), this number seems to be too low. The statement deduced from our literature review, although extreme and by our count too low, should be discussed and be either confirmed or rejected by an expert committee with the aim to define profound minimum volumes. We also outlined the known threats of centralization, namely unjustified exclusion of high-quality surgeons, leaving excluded surgeons and hospitals [11, 13, 30], leaving emergency surgery to low-volume surgeons (LVS) or LVH (excluded from selective surgery), implicating worse experience with surgical oncological treatment of CRC offered to the patients with the highest risk [11], the realistic danger of overload concerning HV [11], and, last but not least, the observation that patients who traveled farther for their care had worse outcomes than expected. This statement, cited in the review by Etzioni et al. [23] and called 'reverse distance bias' in their discussion, may be relevant for the US situation but not necessarily for Western European countries, e.g. Germany. Patients with CC and RC as well as their relatives do want the treatment in a hospital which is easy to reach and which offers the best quality possible.

Our answer to the question 'Do we need minimum volumes for surgeons and/or hospitals?' clearly is 'yes', but the requested minimum volumes should be low if the other high-quality parameters are fulfilled. Treatment by surgeons or in hospitals with too low or no volumes is dangerous as well. There is no doubt that an LVS 
working in or moving to an LVH leads to the worst results [31]. As stated above, a relatively low limit of procedures per year may be set as minimum volumes for surgeons and/or hospitals after ample evaluation and expert discussion of the available literature data. However, we definitely need more objective quality parameters, as these have significant impact on the patients' benefit. In addition to volume alone, besides our list cited above, the following aspects are regarded as important for treatment quality in CC and RC: training and expertise of the surgeons $[1,8,22,23,32,33]$, establishment of multidisciplinary teams $[11-13,23,34]$, and, for monitoring and benchmarking quality, the establishment of audits [12-14, 16, 34]. With these strong statements the discussion of 'volume alone' should be abandoned, as has been previously practiced by German authors/associations $[7,8]$ and by the GCS. The more than 260 LBCC certified by the GCS in 2016 on the basis of S3 guidelines and the recommendations of a structural commission (KHL member of these institutions) are respecting these and other recommendations [24]. The GCS should also respect our current recommendation as to the question 'impact of volume in CC and RC treatment?'. Registration and benchmarking, as practiced e.g. by the DGAV or the WDC (Westdeutsches Darm Centrum), a group simply performing registrations and benchmarking, is better than nothing - but not enough. To obtain a high acceptance, the list of requirements for e.g. 'bowel cancer centers' should not contain too many unproven or low-evidence-level demands. Nowadays, all political decisions on downrating or even excluding hospital units from providing treatment for $\mathrm{CC}$ and or RC must have the highest level of evidence to avoid maltreatment on the one hand and unjustified and disastrous socio-economic consequences by centralization on the other hand. Centralization may have severe disadvantages, such as treatment by surgeons or hospitals who have no interest in providing the respective facts that we are demanding in order to obtain the currently best structure, process and result quality. In conclusion, we naturally agree with the statement of Salz and Sandler [15] (Department of Health Policy and Department of Gastroenterology, respectively, University of North Carolina at Chapel Hill, NC, USA) who had analyzed the effects of HV and SV on the outcome for RC surgery in a review of 23 articles with a total of 5,984,195 patients and stated that 'any effect (of volume), if present, is small'.

\section{Acknowledgement}

This paper is dedicated to Professor Reinhard Bittner for his 75th birthday. He keeps on stimulating his surgical friends from the Ulm Surgical School to provide excellent surgical quality.

\section{Disclosure Statement}

The authors have no conflict of interest.

\section{References}

1 Jafarov S, Kornmann M, Knoefel WT, Link KH: Unterschiede zwischen Kolonkarzinom und Rektumkarzinom. Viszeralmedizin 2016. - 71. Jahrestagung der Deutschen Gesellschaft für Gastroenterologie, Verdauungs- und Stoffwechselkrankheiten 2016;abstract KV 393.

2 Robert Koch-Institut: Bericht zum Krebsgeschehen in Deutschland 2016. Berlin, RKI, 2016, pp 105-111.

3 Guinney J, Dienstmann R, Wang X, et al: The consensus molecular subtypes of colorectal cancer. Nat Med 2015;21:1350-1356.

4 Pox C, Aretz S, Bischoff SC, et al: S3-Leitlinie Kolorektales Karzinom. Version 1.1 - August 2014. AWMFRegisternummer: 021/007OL.

5 Kornmann M, Formentini A, Ette C, Henne-Bruns D, Kron M, Sander S, Baumann W, Kreuser ED, Staib L, Link KH: Prognostic factors influencing the survival of patients with colon cancer receiving adjuvant 5-FU treatment. Eur J Surg Oncol 2008;34:1316-1321.

6 Kornmann M, Staib L, Wiegel T, Kron M, HenneBruns D, Link KH, Formentini A; Study Group Oncology of Gastrointestinal Tumors (FOGT): Long-term results of 2 adjuvant trials reveal differences in chemosensitivity and the pattern of metastases between colon cancer and rectal cancer. Clin Colorectal Cancer 2013 12:54-61.

7 Link KH, Kornmann M, Mann M, Bittner R: Multimodale Therapie von Kolon- und Rektumkarzinomen Qualitätsparameter. Viszeralmedizin 2009;25:105-117.
8 Link KH, Kornmann M, Bittner R, Köckerling F, Arbogast R, Gastinger I, Heitland W, Henne-Bruns D, Lang H, Lippert H, Mann M, Meyer HJ, Polonius MJ, Post S, Raab R, Schiedeck T, Schumpelick V: Qualitätsanforderungen zur Behandlung des Kolon- und Rektumkarzinoms. Chirurg 2010;81:222-230.

9 Kim W, Wolff S, Ho V: Measuring the volume-outcome relation for complex hospital surgery. Appl Health Econ Health Policy 2016;14:453-464.

10 Marusch F, Koch A, Schmidt U, Pross M, Gastinger I, Lippert H: Hospital caseload and the results achieved in patients with rectal cancer. Br J Surg 2001;88:13971402.

11 Rogers SO, Wolf RE, Zaslavsky AM, Wright WE, Ayanian JZ: Relation of surgeon and hospital volume to processes and outcomes of colorectal cancer surgery. Ann Surg 2006;244:1003-1011.

12 van Gijn W, Gooiker GA, Wouters MW, Post PN, Tollenaar RA, van de Velde CJ: Volume and outcome in colorectal cancer surgery. Eur J Surg Oncol 2010; 36(suppl 1):S55-63.

13 Damle RN, Macomber CW, Flahive JM, Davids JS, Sweeney WB, Sturrock PR, Maykel JA, Santry HP, Alavi K: Surgeon volume and elective resection for colon cancer: an analysis of outcomes and use of laparoscopy. J Am Coll Surg 2014;218:1223-1230.

14 Archampong D, Borowski D, Wille-Jørgensen P, Iversen LH: Workload and surgeon's specialty for outcome after colorectal cancer surgery. Cochrane Database Syst Rev 2012;14:CD005391.
15 Salz T, Sandler RS: The effect of hospital and surgeon volume on outcomes for rectal cancer surgery. Clin Gastroenterol Hepatol 2008;6:1185-1193.

16 Kolfschoten NE, Marang-van de Mheen PI, Wouters MW, Eddes EH, Tollenaar RA, Stijnen T, Kievit J; Dutch Surgical Colorectal Audit Group: A combined measure of procedural volume and outcome to assess hospital quality of colorectal cancer surgery, a secondary analysis of clinical audit data. PLoS One 2014; 9:e88737.

17 Pieper D, Eikermann M, Mathes T, Prediger B, Neugebauer EAM: Mindestmengen auf dem Prüfstand. Chirurg 2014;85:121-124.

18 Ruffo G, Barugola G, Rossini R, Sartori CA: Colorectal surgery in Italy. Criteria to identify the hospital units and the tertiary referral centers entitled to perform it. Updates Surg 2016;68:123-128.

19 Buurma M, Kroon HM, Reimers MS, Neijenhuis PA: Influence of individual surgeon volume on oncological outcome of colorectal cancer surgery. Int J Surg Oncol 2015;2015:464570.

20 Liu CJ, Chou YJ, Teng CJ, Lin CC, Lee YT, Hu YW, Yeh CM, Chen TJ, Huang N: Association of surgeon volume and hospital volume with the outcome of $\mathrm{pa}$ tients receiving definitive surgery for colorectal cancer: a nationwide population-based study. Cancer 2015; 121:2782-2790.

21 McArdle CS, Hole DJ: Influence of volume and specialization on survival following surgery for colorectal cancer. Br J Surg 2004;91:610-617. 
22 Hall GM, Shanmugan S, Bleier JI, Jeganathan AN, Epstein AJ, Paulson EC: Colorectal specialization and survival in colorectal cancer. Colorectal Dis 2016;18: 51-60.

23 Etzioni DA, Young-Fadok TM, Cima RR, Wasif N, Madoff RD, Naessens JM, Habermann EB: Patient survival after surgical treatment of rectal cancer: impact of surgeon and hospital characteristics. Cancer 2014;120: 2472-2481.

24 Bruns J: Krebsmedizin zwischen Qualität und Kostendiskussion. Forum 2016;31:484-488.

25 Martling AL, Holm T, Rutqvist LE, Moran BJ, Heald RJ, Cedermark B: Effect of a surgical training programme on outcome of rectal cancer in the County of Stockholm. Stockholm Colorectal Cancer Study Group, Basingstoke Bowel Cancer Research Project. Lancet 2000;356:93-96.

26 Billingsley KG, Morris AM, Dominitz JA, Matthews B, Dobie S, Barlow W, Wright GE, Baldwin LM: Surgeon and hospital characteristics as predictors of major adverse outcomes following colon cancer surgery: understanding the volume-outcome relationship. Arch Surg 2007;142:23-31.
27 Wibe A, Rendedal PR, Svensson E, Norstein J, Eide TJ, Myrvold HE, Søreide O: Prognostic significance of the circumferential resection margin following total mesorectal excision for rectal cancer. Br J Surg 2002;89: 327-334.

28 Urbach DR, Bell CM, Austin PC: Differences in operative mortality between high- and low-volume hospitals in Ontario for 5 major surgical procedures: estimating the number of lives potentially saved through regionalization. CMAJ 2003;168:1409-1414.

29 Halm EA, Lee C, Chassin MR: Is volume related to outcome in health care? A systematic review and methodologic critique of the literature. Ann Intern Med 2002;137:511-520.

30 Rhoads KF, Ackerson LK, Ngo JV, Gray-Hazard FK, Subramanian SV, Dudley RA: Adequacy of lymph node examination in colorectal surgery: contribution of the hospital versus the surgeon. Med Care 2013;51: 1055-1062.

31 Harmon JW, Tang DG, Gordon TA, Bowman HM, Choti MA, Kaufman HS, Bender JS, Duncan MD, Magnuson TH, Lillemoe KD, Cameron JL: Hospital volume can serve as a surrogate for surgeon volume for achieving excellent outcomes in colorectal resection. Ann Surg 1999;230:404-411; discussion 411-413.
32 Ricciardi R, Roberts PL, Read TE, Baxter NN, Marcello PW, Schoetz DJ: Who performs proctectomy for rectal cancer in the United States? Dis Colon Rectum 2011; 54:1210-1215.

33 Smith JA, King PM, Lane RH, Thompson MR: Evidence of the effect of 'specialization' on the management, surgical outcome and survival from colorectal cancer in Wessex. Br J Surg 2003;90:583-592.

34 Breugom AJ, Boelens PG, van den Broek CB, Cervantes A, Van Cutsem E, Schmoll HJ, Valentini V, van de Velde CJ: Quality assurance in the treatment of colorectal cancer: the EURECCA initiative. Ann Oncol 2014;25:1485-1492.

35 Aquina CT, Probst CP, Becerra AZ, Iannuzzi JC, Kelly KN, Hensley BJ, Rickles AS, Noyes K, Fleming FJ, Monson JR: High volume improves outcomes: the argument for centralization of rectal cancer surgery. Surgery 2016;159:736-748.

36 Hermanek P, Wiebelt H, Staimmer D, Riedl S: Prognostic factors of rectum carcinoma-experience of the German Multicenter Study SGCRC. German Study Group Colo-Rectal Carcinoma. Tumori 1995;81(suppl): 60-64. 